\section{Relação entre sentido de vida e espiritualidade na América Latina: uma revisão integrativa da literatura}

\author{
Andreia Barbosa da Silva \\ Valeschka Martins Guerra \\ Gustavo Pfister Pirola \\ Jussara Abilio Galvão \\ Lilian Gazzoli Zanotelli
}

\section{RESUMO}

Este estudo visa investigar a produção científica em Psicologia acerca da relação entre sentido de vida e espiritualidade, no contexto da América Latina. Realizou-se uma busca por artigos científicos publicados em periódicos nacionais e internacionais, indexados nas plataformas eletrônicas DOAJ, SciELO e Redalyc, os quais contivessem os termos "sentido de vida" e "espiritualidade" ou "religiosidade", em espanhol, inglês e português, publicados de 2007 a 2017. Foram excluídos artigos cujo texto completo não foi disponibilizado; que não apresentaram os descritores em qualquer parte do texto; e títulos duplicados. Os dados dos artigos selecionados foram registrados em planilha eletrônica, sendo submetidos a análises descritivas. Das referências recuperadas nas bases eletrônicas $(\mathrm{N}=3.009)$, apenas 19 artigos mostraram-se pertinentes ao estudo proposto. Os resultados apontaram que existe um baixo número de publicações correlacionando os construtos na Psicologia, indicando um campo de investigação a ser explorado, principalmente em relação à adaptação e validação de instrumentos psicométricos para mensuração do construto espiritualidade.

Palavras-chave: sentido de vida; espiritualidade; revisão de literatura; América Latina.

\section{ABSTRACT}

\section{Relation between the meaning of life and spirituality in Latin America: An integrative literature review}

This study aims at investigating the scientific production in Psychology about the relation between meaning of life and spirituality in the context of Latin America. Scientific articles published in national and international journals, indexed in the electronic platforms DOAJ, SciELO and Redalyc were searched, containing the terms "meaning in life" and "spirituality" or "religiosity" in Spanish, English, and Portuguese, published from 2007 to 2017. Exclusion criteria included articles with no full text available, papers that did not present the descriptors in any part of the text or not related to each other, and duplicate titles. Data of the selected articles were recorded in an electronic spreadsheet and submitted to descriptive analyzes. Of the references retrieved from electronic databases $(N=3,009)$, only 19 articles were pertinent to the proposed study. Results indicated that there is a low number of publications correlating the constructs in Psychology, indicating a field of investigation to be explored, mainly in relation to the adaptation and validation of psychometric instruments for measurement of the spirituality construct.

\section{Sobre os Autores}

A. B. S. https://orcid.org/0000-00019112-6044 Universidade Federal do Espírito Santo, Vitória - ES andreia.silva@ufes.br

V. M. G. http://orcid.org/0000-0001-7455$125 \mathrm{X}$ Universidade Federal do Espírito Santo), Vitória - ES valeschka.guerra@ufes.br

G. P. P.

https://orcid.org/0000-00018522-5679

Universidade Federal do Espírito Santo), Vitória - ES gustavopfister@hotmail.com

J. A. G.

http://orcid.org/0000-0003-48831113

Universidade Federal do Espírito Santo), Vitória - ES

jussaraabgalvao@hotmail.com

L. G. Z.

https://orcid.org/0000-0002-

4869-1504

Universidade Federal do Espírito Santo), Vitória - ES

liliangazzoli@yahoo.com.br

\section{Direitos Autorais}

Este é um artigo aberto e pode ser reproduzido livremente, distribuído, transmitido ou modificado, por qualquer pessoa desde que usado sem fins comerciais. 0 trabalho é disponibilizado sob a licença Creative Commons CC-BY-NC

Keywords: meaning of life; spirituality; literature review; Latin America. 
A discussão acerca do sentido de vida vem apresentando uma evolução considerável, em relação a sua definição e instrumentos de mensuração. Esse fenômeno tornou-se objeto de estudo de diferentes áreas de conhecimento, em contextos variados, sendo associado a variáveis diversas, como: construtos psicológicos (bem-estar, qualidade de vida, emoções positivas em situações de doenças, nas crises e nas adversidades, dentre outros); e/ou dados sociodemográficos (Damásio \& Koller, 2016; Marcelino, 2018; Rodrigues, 2015).

No campo da Psicologia, o interesse em compreender o que seria o sentido da vida foi influenciado por reflexões filosóficas existencialistas, sendo a Psicologia Humanista a corrente psicológica responsável pela propagação da ideia de que o construto sentido de vida seria um componente fundamental para o funcionamento psicológico positivo. Diferentemente das abordagens filosóficas e teológicas, a corrente humanista originou uma perspectiva de que cada sujeito teria (ou deveria ter) um sentido único e exclusivo para sua própria existência. Dentro dessa linha, destacam-se autores tradicionais, como: Gordon Allport, Abraham Maslow, Carl Rogers e, principalmente, Viktor Frankl (Damásio \& Koller, 2016).

Os estudos acerca do sentido de vida foram impulsionados pelos pressupostos teóricos de Viktor Frankl, que inaugurou uma escola de psicoterapia denominada Logoterapia e Análise Existencial, conhecida como a psicoterapia centrada no sentido da vida (Aquino et al., 2009). Em sua teoria, o autor argumentou que a motivação primordial do ser humano é a busca por sentido de vida, que se refere ao interesse contínuo por um significado para a vida (Frankl, 2016, 2018).

A perspectiva de Frankl concebe a busca de sentido como um fenômeno originário da dimensão espiritual do ser humano. Além de preocupar-se com as realidades existenciais da psicologia humana e com a sua motivação primária por sentido (vontade de sentido), essa abordagem está orientada pela conscientização da importância da dimensão espiritual na experiência humana, considerando-a como essência da existência, integrada às demais dimensões do indivíduo, quais sejam: biológica, psicológica e social (Frankl, 1993; Coelho \& Mahfoud, 2001; Silva \& Silva, 2014).

Apesar da teoria de Frankl estar pautada no sentido de vida (SV), o autor não se prendeu a uma definição do construto (Damásio \& Koller, 2016). A partir dos pressupostos de Frankl, outros autores tentaram sistematizar o SV. Para fins deste artigo, será adotada a concepção encontrada no estudo de Damásio et al. (2013), a qual de- fine o construto como percepção de ordem e coerência na própria existência, aliada à busca e ao cumprimento de objetivos significativos, que resultam na sensação de realização e/ou felicidade, com base nas proposições de Reker e Wong (1988) e Steger (2009).

Além do mais, estudos vêm apontando a busca de sentido por meio da espiritualidade, com ou sem a adesão a uma religião específica (Cavalheiro \& Falcke, 2014; Coelho \& Mahfoud, 2001; Costa et al., 2008; Marques \& Aguiar, 2014; Marques et al., 2009; Oliveira \& Aquino, 2014). Nesse contexto, Barros-Oliveira (2007), afirma que a espiritualidade pode ser definida como busca de significado e de objetivos na vida relacionados com o sagrado ou o transcendente, incluindo aspectos ou elementos cognitivos, afetivos e experienciais.

Na literatura científica, é comum encontrar o termo espiritualidade relacionado ao conceito de religião, encontrando teorias e pressupostos diversos que interferem em uma definição consensual acerca da espiritualidade no campo da Psicologia. As concepções sobre a espiritualidade, ao que tudo indica, apontam para um conceito abrangente, envolvendo busca pelo sagrado, sentido na vida, transcendência e desenvolvimento do potencial humano; ao contrário da religião, que está sendo mais associada a uma instituição ideológica, de práticas religiosas formais. Nesse sentido, a vivência de uma religião seria apenas uma das formas possíveis de busca pelo sagrado/transcendente (Koenig et al., 2012; Pargament, 2009; Zinnbauer \& Pargament, 2005).

Em estudo realizado no Brasil, Moreira-Almeida et al. (2006) conceituaram a espiritualidade como uma busca pessoal de compreensão relacionada a questões existenciais maiores e suas relações com o sagrado e/ou transcendente. Esse conceito de espiritualidade pode (ou não) levar ao desenvolvimento de práticas religiosas ou na formação de comunidades religiosas. A religião, nesse sentido, seria um sistema organizado de crenças, práticas, rituais e símbolos destinados a facilitar a proximidade com o sagrado ou transcendente (Deus, poder superior ou verdade / realidade última). Outras publicações brasileiras vêm empregando essa diferenciação na concepção dos termos, inclusive na sua mensuração (Braghetta, 2017; Marques \& Aguiar, 2014).

Cabe destacar que o Brasil apresenta um alto nível de envolvimento religioso. Segundo estudo realizado por, Moreira-Almeida et al. (2010), 95\% da população brasileira tem uma religião, $83 \%$ consideram religião muito importante e $37 \%$ frequentam serviços religiosos pelo menos uma vez por semana. Marques et al. (2009) indi- 
caram a importância de estudos em estados brasileiros acerca da espiritualidade, assim como alertaram sobre o enorme potencial cristão e religioso da população da América Latina, que pode ser um campo de investigação com contribuições importantes para pesquisas que envolvam o tema espiritualidade/religiosidade.

Considerando os processos de transformação relacionados às crenças religiosas, seus conceitos e impactos, assim como o crescimento do envolvimento religioso, Marques et al. (2009) apontaram a necessidade de investigação e de medidas para melhor captar as qualidades dinâmicas da religião e da espiritualidade. Neste sentido, destaca-se, principalmente, a importância da criação, validação e adaptação de escalas que mensurem apenas o fenômeno da espiritualidade, visto que a maioria das medidas se concentra na religiosidade e não na espiritualidade (Sherman \& Simonton, 2001).

Diante de todo o exposto, é perceptível a existência de um campo de possibilidades a ser explorado na correlação entre o sentido de vida e a espiritualidade, no contexto da América Latina e, principalmente, do Brasil, de modo a contribuir para a ampliação do conhecimento dessa temática na literatura. Assim, o objetivo deste artigo foi realizar uma revisão integrativa da literatura na área da Psicologia na América Latina, no intuito de verificar as abordagens mais utilizadas nos estudos, metodologias, as possíveis lacunas e as contribuições para a compreensão desses fenômenos.

\section{MÉTODO}

A revisão integrativa da literatura foi desenvolvida utilizando-se os mesmos procedimentos de uma revisão sistemática, por serem processos de investigação que permitem a reunião, avaliação crítica e síntese de resultados de múltiplos estudos (Costa \& Zoltowski, 2014). A diferença entre os métodos localiza-se no tipo de pergunta de pesquisa e no tipo de material que se busca: a revisão sistemática direciona seu levantamento para pesquisas com resultados já publicados, de forma a garantir um levantamento das evidências já existentes acerca de uma determinada pergunta de pesquisa, enquanto que a revisão integrativa busca levantar o corpo de conhecimento existente sobre um determinado tema, mesmo que este não envolva resultados de pesquisas empíricas (Ercole et al., 2014). Esse método sistematizado de busca de artigos foi adotado para mapear a produção científica acerca dos temas na área da Psicologia no contexto da América Latina.

\section{FONTES DE DADOS}

Foi realizada uma busca por artigos de revisão, teóricos e empíricos publicados em periódicos brasileiros e internacionais, indexados nas bases de dados: SciELO (Scientific Eletronic Library Online), DOAJ (Directory of Open Access Journals); e Redalyc (Red de Revistas Científicas de América Latina y el Caribe, España y Portugal). As fontes eletrônicas de dados foram escolhidas por disponibilizar textos completos e acesso gratuito, bem como concentrar as produções científicas da América Latina.

\section{PROCEDIMENTOS DE COLETA}

Como estratégia de localização dos artigos nas bases de dados, foram consideradas as variáveis norteadoras do estudo (sentido de vida e espiritualidade), utilizando os descritores em três idiomas (português, inglês e espanhol) e operadores booleanos, a saber: "sentido de vida"/ "meaning in life"/ "sentido de la vida" AND (espiritualidade OR religiosidade/ spirituality OR religiosity/ espiritualidad OR religiosidad). Apesar de apresentar usos e significados diferentes, os termos "espiritualidade" e "religiosidade" foram ambos utilizados de modo a recuperar maior diversidade de artigos acerca da temática proposta.

0 processo de seleção e armazenamento dos artigos foi realizado em junho de 2018, por dois juízes independentes, obedecendo aos seguintes critérios de inclusão e exclusão: a) ano de publicação (de 2007 a 2017); b) idiomas (português, inglês e espanhol); c) área de conhecimento (Psicologia); d) local de produção (América Latina); e) artigos que apresentarem, simultaneamente, os descritores estabelecidos em qualquer parte do texto; f) disponibilização de texto completo; g) apenas artigos (não incluir resenhas de livros e resumos de dissertações e teses); e h) artigos que apresentam os termos sentido de vida e espiritualidade/religiosidade diretamente correlacionados.

\section{ORGANIZAÇÃO E TRATAMENTO DE DADOS}

Os artigos selecionados foram registrados em uma planilha eletrônica, catalogados por: a) título do artigo; b) autor(es); c) palavras-chave; d) ano de publicação; e) periódico; f) classificação Qualis; g) país de realização da pesquisa; $h$ ) tipo de artigo; i) resumo; j) objetivos; $k$ ) população; I) tamanho da amostra; $m$ ) métodos de coleta; $n$ ) variáveis estudadas; o) método de análise; $p$ ) principais resultados; e q) recomendações/conclusões dos autores. A avaliação dos artigos catalogados foi realizada por dois 
juízes, havendo manifestação de um terceiro juiz nos casos de discordância. Os dados do banco final foram organizados, tabulados e submetidos à análise de frequência, porcentagem e de conteúdo.

\section{RESULTADOS E DISCUSSÃO}

$\mathrm{Na}$ busca inicial foram recuperadas 3.009 referências (DOAJ N = 206; SciElo N = 25; e Redalyc N = 2.778). Desse total, foram excluídas 2.589 referências por não atender aos critérios de inclusão relacionados ao ano de publicação, ao idioma e à área de conhecimento. Registra-se que $78 \%$ (setenta e oito por cento) das publicações excluídas, nessa busca inicial, ocorreram por não pertencer à área da Psicologia, sendo selecionadas 420 referências.

Das 420 referências selecionadas, foram excluídos 04 trabalhos cujos textos completos não estavam disponibilizados nas bases eletrônicas; e 102 estudos não produzidos por países da América Latina, restando 314 refe- rências potencialmente relevantes. Em seguida, os juízes realizaram busca, dentre essas referências, por artigos que apresentassem, simultaneamente, os descritores estabelecidos em qualquer parte do texto, sendo também removidos os documentos duplicados, permanecendo 57 artigos. Após análise de conteúdo, por parte dos juízes, 38 artigos foram excluídos por não apresentar os termos da pesquisa diretamente correlacionados.

Os achados no processo de busca e seleção dos artigos evidenciaram que, no contexto da América Latina, existe um baixo número de publicações científicas em Psicologia correlacionando os termos sentido de vida e espiritualidade. Das 3.009 referências recuperadas nas bases eletrônicas mencionadas, apenas 19 artigos mostraram-se pertinentes ao estudo proposto (tais artigos encontram-se citados na lista de referências precedidos por um asterisco). A seguir, demonstra-se, resumidamente, o processo de busca e seleção dos artigos, por meio da Figura 1.

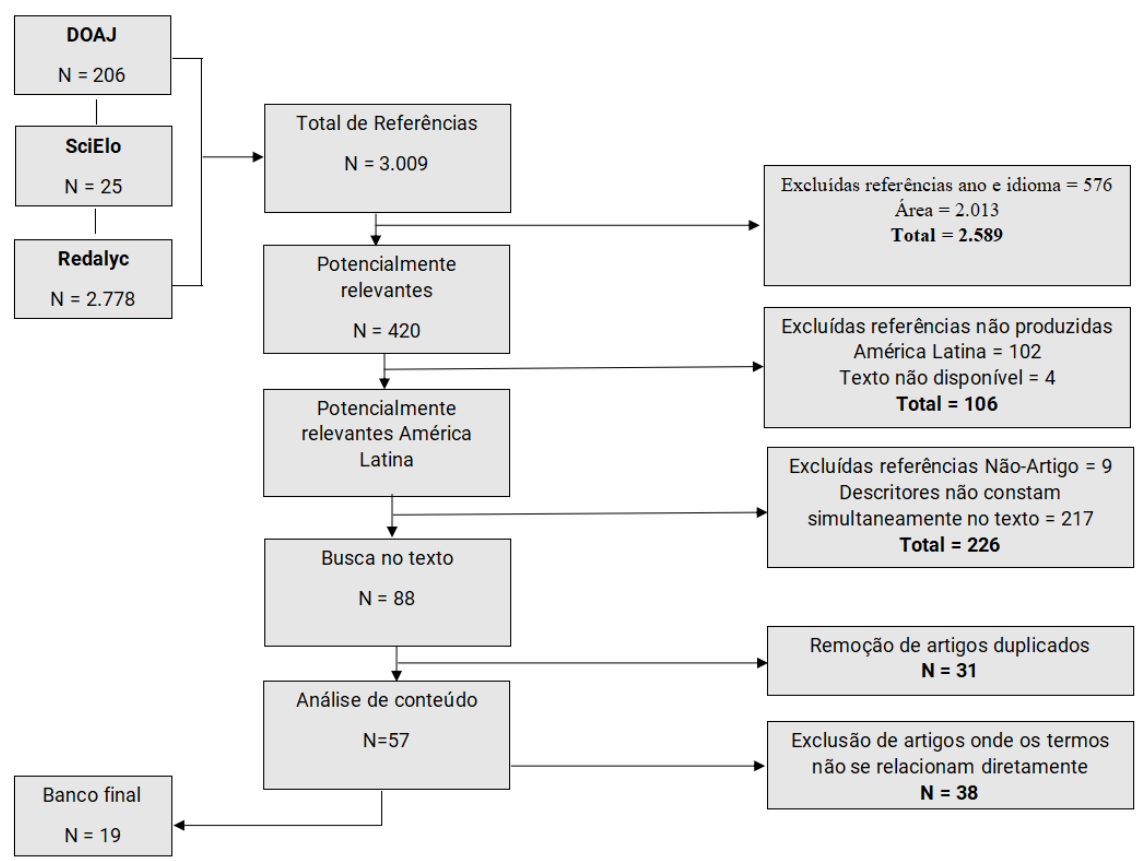


Quanto aos aspectos gerais, conforme dados relatados na Tabela 1, os artigos selecionados, em sua maioria, foram produzidos no Brasil, e o número de publicações, a cada ano, manteve-se relativamente constante, registrando-se aumento na produção apenas nos anos de 2013 e 2016. Em relação aos tipos de estudos realizados, houve um destaque significativo para os Relatos de Pesquisa, que representam cerca de $80 \%$ dos trabalhos analisados, apontando uma baixa produção em termos de Relato de Intervenção, Ensaio Teórico e Revisão de Literatura. A falta de artigos teóricos pode evidenciar a necessidade de geração e avaliação de conhecimento acerca dos fenômenos propostos, ou seja, necessidade desses fe- nômenos serem observados e testados empiricamente, para viabilizar, posteriormente, a explicação por parte dos pesquisadores, na forma de teoria.

Verificou-se ainda que a maior parte dos periódicos publicou apenas um artigo sobre a temática, sinalizando a dispersão das publicações. No que diz respeito à qualidade dos artigos, segundo a última Tabela de Avaliação Trienal da Capes (2017), os trabalhos foram publicados em revistas avaliadas entre os estratos $A 1$ e $B 2$, sendo que $58,8 \%$ das produções foram classificadas no estrato A2.

Tabela 1. Distribuição dos artigos por Ano, Tipo, Periódico, conceito Qualis e Pais de Publicação

\begin{tabular}{lcc}
\hline Variável & $\mathrm{F}$ & $\%$ \\
\hline Ano de publicação & & \\
\hline 2008 & 1 & $5,3 \%$ \\
2009 & 1 & $5,3 \%$ \\
2010 & 2 & $10,5 \%$ \\
2011 & 1 & $5,3 \%$ \\
2012 & 2 & $10,5 \%$ \\
2013 & 3 & $15,8 \%$ \\
2014 & 1 & $5,3 \%$ \\
2015 & 2 & $10,5 \%$ \\
2016 & 4 & $21,1 \%$ \\
2017 & 2 & $10,5 \%$ \\
\hline Tipo de Estudo & & \\
\hline Relato de Pesquisa & 15 & $78,9 \%$ \\
Revisão de Literatura & 2 & $10,5 \%$ \\
Relato de Intervenção & 1 & $5,3 \%$ \\
Ensaio Teórico & 1 & $5,3 \%$ \\
\hline Periódicos & & \\
\hline Universitas Psychologica & 2 & $10,5 \%$ \\
Revista Argentina de Clínica Psicológica & 2 & $10,5 \%$ \\
Temas em Psicologia & 2 & $10,5 \%$ \\
Psicologia: Ciência e Profissão & 1 & $5,3 \%$ \\
Acta Colombiana de Psicologia & 1 & $5,3 \%$ \\
Liberabit-Revista De Psicologia & 1 & $5,3 \%$ \\
\hline
\end{tabular}




\begin{tabular}{lcc} 
Psico-USF & 1 & $5,3 \%$ \\
Estudos de Psicologia (Campinas) & 1 & $5,3 \%$ \\
Psicologia \& Sociedade & 1 & $5,3 \%$ \\
Estudos e Pesquisas em Psicologia & 1 & $5,3 \%$ \\
Psicología Iberoamericana & 1 & $5,3 \%$ \\
Arquivos Brasileiros de Psicologia & 1 & $5,3 \%$ \\
Estudos de Psicologia & 1 & $5,3 \%$ \\
Psicologia: Reflexão e Crítica & 1 & $5,3 \%$ \\
Acta de Investigación Psicológica & 1 & $5,3 \%$ \\
Boletim da Academia Paulista de Psicologia & 1 & $5,3 \%$ \\
\hline Qualis (Obs: Não constava o conceito Qualis de dois artigos) & & \\
\hline A2 & 10 & $58,8 \%$ \\
A1 & 5 & $29,4 \%$ \\
B1 & 1 & $5,9 \%$ \\
B2 & 1 & $5,9 \%$ \\
\hline País de Publicação & & \\
\hline Brasil & 12 & $63,2 \%$ \\
Colômbia & 3 & $15,8 \%$ \\
Chile & 1 & $5,3 \%$ \\
México & 1 & $5,3 \%$ \\
Argentina & 1 & $5,3 \%$ \\
Peru & 1 & $5,3 \%$ \\
\hline
\end{tabular}

Os dados demonstrados indicam que os estudos foram apresentados em revistas com um padrão de qualidade satisfatório. Revelam, ainda, que a população da América Latina é um campo de investigação pouco explorado, e as pesquisas encontradas estão voltadas preponderantemente para a população brasileira.

Essa população estudada nos artigos apareceu de forma diversificada, sendo, majoritariamente, representada por homens e mulheres (31,57\%); seguida por estudos com idosos $(15,80 \%)$ e com pacientes $(15,80 \%)$. Os demais artigos possuíam amostras representadas por populações esporádicas, como se mostra na Tabela 2. Nes- ses trabalhos, o tamanho da amostra variou da seguinte forma: cinco artigos apresentaram até 20 participantes (26,32\%); cinco artigos com amostra entre 101 e 1000 participantes $(26,32 \%)$; três artigos obtiveram amostras entre 51 e 100 participantes (15,79\%); e apenas um artigo com amostra entre 1001 e 5000 participantes (5,26\%). Cabe ressaltar que quatro artigos selecionados não envolveram a participação de sujeitos em razão do tipo de estudo adotado, qual seja: revisão de literatura (Santos, 2016; Sommerhalder, 2010); ensaio teórico (Moreira \& Holanda, 2010) e estudo documental (Florenzano et al., 2015). 
Tabela 2: Distribuição das publicações de acordo com a população estudada

\begin{tabular}{|c|c|c|c|c|}
\hline População & Autor(es) & $\begin{array}{l}\text { Faixa } \\
\text { etária }\end{array}$ & Média (DP) & $\%$ por sexo \\
\hline \multirow{6}{*}{$\begin{array}{l}\text { Homens e } \\
\text { Mulheres }\end{array}$} & Aquino et al. (2009) & 18 a 84 & $42(16,7)$ & $37 \% \mathrm{H} / 63 \% \mathrm{M}$ \\
\hline & Assis, Faria e Lins (2014) & 21 a 78 & $52,6(12,5)$ & $26 \% \mathrm{H} / 74 \% \mathrm{M}$ \\
\hline & Damásio, Koller e Schnell (2013) & 18 a 91 & $33,9(15,0)$ & $36 \% \mathrm{H} / 64 \% \mathrm{M}$ \\
\hline & Núñez-Rodriguez, Souza e Koller (2016) & 18 a 30 & $22,8(3,4)$ & $43 \% \mathrm{H} / 57 \% \mathrm{M}$ \\
\hline & Parra (2008) & 18 a 76 & $45,94(13,3)$ & $27 \% \mathrm{H} / 73 \% \mathrm{M}$ \\
\hline & Parra e Corbetta (2013) & 21 a 71 & $43,92(12,0)$ & $44 \% \mathrm{H} / 66 \% \mathrm{M}$ \\
\hline \multirow{3}{*}{ Idosos } & Estrada, Fortaleza, Garcia e Palos (2012) & 68 & - & $31 \% \mathrm{H} / 69 \% \mathrm{M}$ \\
\hline & Melo e Araújo (2013) & 63 a 93 & 74,8 & $35 \% \mathrm{H} / 65 \% \mathrm{M}$ \\
\hline & Vieira e Aquino (2016) & 60 a 87 & $67,2(5,6)$ & $10 \% \mathrm{H} / 90 \% \mathrm{M}$ \\
\hline \multirow{3}{*}{ Pacientes } & Barbosa, Matamoros e Pedraza (2015) & maior 18 & - & $100 \% \mathrm{M}$ \\
\hline & Benites, Neme e Santos (2017) & 44 a 71 & - & $50 \% \mathrm{H} / 50 \% \mathrm{M}$ \\
\hline & Payán, Vinaccio e Quiceno (2011) & 21 a 78 & $52,6(12,5)$ & $26 \% \mathrm{H} / 74 \% \mathrm{M}$ \\
\hline Crianças & Becker e Silva (2016) & 6 a 10 & - & $50 \% \mathrm{H} / 50 \% \mathrm{M}$ \\
\hline Psicólogos & Oliveira e Junges (2012) & - & - & - \\
\hline Estudantes & Simkin (2017) & 19 a 55 & $25,16(6,0)$ & $43 \% \mathrm{H} / 63 \% \mathrm{M}^{*}$ \\
\hline Total & 15 & $100 \%$ & & \\
\hline
\end{tabular}

* Nota. Soma superior a $100 \%$, conforme citado no artigo original.

As pesquisas apontadas na Tabela 2 indicaram a importância de estudos acerca do sentido de vida e/ou a espiritualidade/religiosidade nas populações investigadas. Verifica-se que todas as faixas etárias apareceram nas pesquisas, sendo estudadas, especificamente, enquanto etapa de desenvolvimento, as faixas etárias: a) idosos; e b) infância. Na pesquisa bibliográfica exploratória de Sommerhalder (2010), apesar de não envolver a participação de sujeitos, a autora abordou acerca do sentido de vida na fase adulta e velhice, no contexto de adaptações inerentes ao processo do envelhecimento. No estudo, foi destacada a relevância de investigar-se como as fontes de sentido da vida se apresentam e são notadas nessas etapas do desenvolvimento humano, com vistas ao me-
Ihor entendimento sobre a atribuição de sentido de vida ao longo da trajetória dos indivíduos.

Acerca do delineamento metodológico dos estudos, conforme demonstrado na Tabela 3, prevaleceu a abordagem quantitativa, com utilização de instrumentos de coleta de dados voltados para esse tipo de pesquisa, quais sejam: escalas, questionários e inventários. Nos estudos qualitativos, foram adotadas, predominantemente, entrevistas (semiestruturadas e fenomenológica). Também houve coleta de dados por meio de revisão de literatura, revisão sistemática, desenho temático em atividade grupal de intervenção e ensaio teórico. 
Tabela 3. Distribuição das publicações de acordo com o tipo de estudo e a coleta de dados

\begin{tabular}{lllll}
\hline Tipo de Pesquisa & \multicolumn{2}{l}{ Artigos } & \multicolumn{2}{l}{ Método } \\
\hline Abordagem & Quanti & 8 & $(\%)$ & Coleta \\
& Quali & 4 & 21,05 & Entrevistas \\
Relato de Pesquisa & Mista & 3 & 15,79 & Escalas; Inventários; Entrevista semiestrutu- \\
& Intervenção & 1 & 5,26 & Revisão de Literatura; Atividades Grupais \\
Revisão de Literatura & & 2 & 10,53 & Revisão Sistemática \\
Ensaio Teórico & & 1 & 5,26 & Revisão de Literatura \\
\hline Total & & 19 & 100,00 & \\
\hline
\end{tabular}

Das abordagens metodológicas encontradas, identifica-se a propensão de busca por evidências científicas através da testagem de teorias objetivas, examinando a relação de variáveis estabelecidas nos estudos. Registram-se estudos de natureza qualitativa, o que pode evidenciar o esforço em investigar com maior profundidade as percepções e significados acerca dos fenômenos propostos em diferentes circunstâncias de vida dos indivíduos. Contudo, existe uma carência de pesquisas de natureza teórica, as quais poderiam problematizar e explicar melhor a correlação entre eles.

$\mathrm{Na}$ variedade de métodos de pesquisa adotados, foram utilizados instrumentos psicométricos, validados pela literatura e/ou adaptados para os estudos, com fins de avaliação dos construtos, sendo encontrados: cinco questionários que avaliaram o construto sentido de vida; quatro escalas, inventários e/ou questionários que mensuraram o construto espiritualidade; duas escalas que avaliaram o construto religiosidade; e duas escalas que avaliaram o construto espiritualidade e o construto religiosidade, segundo Tabela 4.

Dos instrumentos utilizados para investigar o construto sentido de vida, encontram-se adaptados e validados ao contexto brasileiro: a) Questionário de Sentido de Vida (QSV), aplicado no estudo de Vieira e Aquino (2016); b)
Questionário de Fontes de Significado e Sentido na Vida (SoMe-BR), realizada no estudo de Damásio et al. (2013); c) Questionário ou Teste de propósito de vida (PIL-Test), adotado nos estudos de Aquino et al. (2009) e Parra e Corbetta (2013); d) Questionário The Aspiration Index, desenvolvida no estudo de Núñez-Rodriguez et al. (2016). Com exceção do The Aspiration Index, esses instrumentos avaliam aspectos existenciais humanos relacionados ao conceito sentido de vida adotado no presente estudo, segundo Damásio et al. (2013) e Damásio e Koller (2016), abordando índice de sentido de vida, busca por sentido e fontes de sentido, a partir dos pressupostos da Logoterapia e Análise Existencial.

O The Aspiration Index foi utilizado para avaliar diferentes tipos de metas de vida (sucesso financeiro, imagem, popularidade, autoaceitação, saúde física, afiliação, sensação de comunidade, espiritualidade, conformidade, hedonismo e segurança). Apesar de ser pautado na Teoria da Autodeterminação, foi indicado por Núñez-Rodriguez et al. (2016) para beneficiar pesquisas que avaliam o construto sentido de vida com a população brasileira de diferentes regiões e níveis socioeconômicos. Já o Questionário de Experiências de Vida foi desenvolvido no estudo de Parra (2008), na Argentina, e não foi localizada a versão desse instrumento para a população brasileira. 
Tabela 4. Distribuição dos instrumentos utilizados nos artigos selecionados

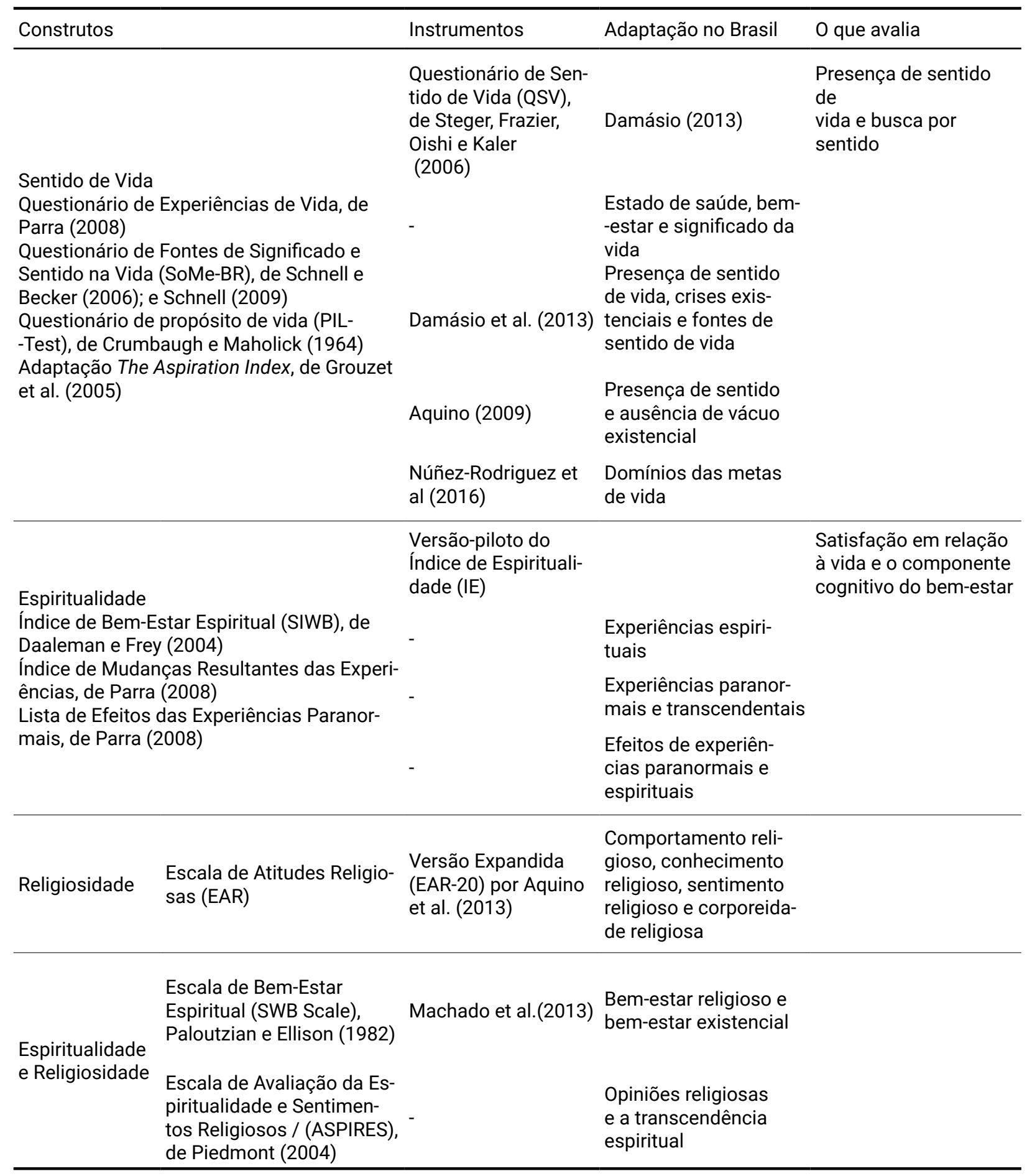


Quanto aos instrumentos de mensuração do construto espiritualidade, não foram encontradas adaptações e validações no Brasil. A Versão-piloto do Índice de Espiritualidade (IE), realizada por Estrada et al. (2012), no Méxi$\mathrm{co}$, avalia o bem-estar espiritual frente à satisfação com a vida. O Índice de Bem-Estar Espiritual (SIWB) foi aplicado no estudo de Payán et al. (2011), na Colômbia, contendo as seguintes dimensões: autoeficácia e esquemas de vida. O Índice de Mudanças Resultantes das Experiências e a Lista de Efeitos das Experiências Paranormais foram desenvolvidos no estudo de Parra (2008), na Argentina, e foram utilizadas por Parra e Corbetta (2013), em estudo realizado no Peru.

Os instrumentos adotados para investigar o construto religiosidade foram desenvolvidos a partir de estudos brasileiros (Aquino, 2009; Aquino et al., 2013). A Escala de Atitude Religiosa/Espiritualidade foi elaborada originalmente em português, avalia atividades relacionadas aos componentes da atitude, afetivo comportamental e cognitivo. A Versão Expandida da Escala de Atitudes Religiosas (EAR-20), validada em português, é composta por quatro dimensões: comportamento religioso, conhecimento religioso, sentimentos religiosos e corporeidade religiosa.

Além destes, foram encontradas duas escalas que avaliaram tanto o construto espiritualidade quanto 0 construto religiosidade: a) Escala de Bem-Estar Espiritual (SWB Scale), Paloutzian e Ellison (1982), adaptada e validada no Brasil por Machado et al. (2013), que avalia as dimensões: bem-estar religioso e bem-estar existencial; e b) Escala de Avaliação da Espiritualidade e Sentimentos Religiosos (ASPIRES), de Piedmont (2004), adaptada e validada ao contexto espanhol, não tendo versão adaptada ao Brasil. Essa escala avalia sentimentos religiosos e transcendência espiritual, sendo que o primeiro mensura aspectos relacionados à participação religiosa e aos conflitos religiosos. A segunda dimensão (transcendência espiritual), compreende aspectos ligados ao cumprimento da oração, conectividade espiritual e universalidade.
Vale ressaltar que os instrumentos utilizados para mensurar espiritualidade e religiosidade avaliam aspectos específicos voltados a questões religiosas, assim como a espiritualidade de forma mais global, apontando diferenciação dos conceitos conforme encontrado na literatura. Contudo, verificou-se que há uma carência de mensuração do construto espiritualidade adaptado e validado ao contexto brasileiro. Os instrumentos de mensuração utilizados nos estudos brasileiros apresentam-se ligados, principalmente, por aspectos vinculados ao sentido de vida e religiosidade.

Além das abordagens conceituais e/ou teóricas encontradas nos instrumentos adotados, a partir de levantamento dos conteúdos dos artigos, foram constatados 26 conceitos e teorias, conforme Tabela 5. Na análise dos conceitos e teorias apresentados, o termo bem-estar apareceu de forma muito frequente, relacionado ao bem-estar subjetivo ou bem-estar psicológico (Assis et al. 2014; Estrada et al., 2012; Parra, 2008; Parra \& Corbetta, 2013); ou o bem-estar espiritual (Barbosa et al., 2015; Melo \& Araújo, 2013; Payán et al. 2011). Nesses estudos, percebe-se que o sentido de vida e a espiritualidade e/ou religiosidade são elementos integrantes do bem-estar e da qualidade de vida. Isso pode indicar a influência desses construtos para um funcionamento psicológico saudável.

A Logoterapia e Análise Existencial, por sua vez, foi a teoria mais empregada, dentre os artigos selecionados (Aquino et al., 2009; Moreira \& Holanda, 2010; Santos, 2016; Sommerhalder, 2010; Vieira \& Aquino, 2016). Percebe-se o interesse no desenvolvimento dessa abordagem, tanto que atualmente existem 130 grupos de estudo no mundo credenciados à Associação Internacional de Logoterapia e Análise Existencial, sendo que 27\% desses grupos estão localizados em países latino-americanos (Viktor Frankl Institut, 2018). Segundo estudo de Véras e Rocha (2014), a Logoterapia está em fase de ampliação no Brasil, apresentando objetos de estudo relevantes para profissionais de diversas áreas. 
Tabela 5. Distribuição dos conceitos e teorias identificados nos estudos selecionados

\begin{tabular}{lcclcc}
\hline Conceitos e teorias & $\mathrm{N}$ & $\%$ & Conceitos e teorias & $\mathrm{N}$ & $\%$ \\
\hline Bem-estar & 7 & 15,91 & Sofrimento & 1 & 2,27 \\
Logoterapia e Análise existencial & 5 & 11,36 & Resiliência & 1 & 2,27 \\
Velhice & 4 & 9,10 & Mapa de conceitos & 1 & 2,27 \\
Câncer & 3 & 6,82 & Morte/finitude & 1 & 2,27 \\
Qualidade de vida & 2 & 4,56 & Saúde mental & 1 & 2,27 \\
Experiências paranormais & 2 & 4,56 & Prática do psicólogo & 1 & 2,27 \\
Atitude religiosa & 2 & 4,56 & Fase adulta & 1 & 2,27 \\
Suicidalidade & 1 & 2,27 & Cuidados paliativos & 1 & 2,27 \\
Modelo dos cinco fatores & 1 & 2,27 & Fenomenologia & 1 & 2,27 \\
Cognição & 1 & 2,27 & Fontes de sentido & 1 & 2,27 \\
Metas & 1 & 2,27 & Psicologia analítica & 1 & 2,27 \\
Infância & 1 & 2,27 & Vitalidade subjetiva & 1 & 2,27 \\
Teoria da autodeterminação & 1 & 2,27 & Transtorno afetivo & 1 & 2,27 \\
\hline (Continuação) & & & Total & 44 & $100 \%$ \\
\hline
\end{tabular}

Cabe ressaltar que foram encontradas outras teorias nos estudos, como: o Modelo dos cinco fatores (Simkin, 2017), a Teoria da autodeterminação (Núñez-Rodriguez et al., 2016), a Fenomenologia (Benites et al., 2017) e a Psicologia analítica (Mello \& Araújo, 2013). Contudo essas abordagens foram empregadas apenas uma vez. É oportuno frisar que, dos 19 estudos selecionados, apenas oito fundamentaram-se em teorias, o que pode indicar a falta de apropriação de um corpo teórico que fundamente as pesquisas sobre o sentido de vida e/ou espiritualidade/ religiosidade.

Ademais, foi identificado que os fenômenos sentido de vida e espiritualidade são estudados em circunstâncias difíceis da vida, que exigem o enfrentamento de adversidades, ocasiões em que ocorre um questionamento existencial, quais sejam: velhice (Estrada et al., 2012; Mello \& Araújo, 2013; Sommerhalder, 2010; Vieira \& Aquino, 2016); doença terminal, no caso, o câncer (Barbosa et al., 2015; Benites et al., 2017; Payán et al., 2011); suicidalidade e transtornos afetivos (Florenzano et al., 2015); sofrimento e morte/finitude (Moreira \& Holanda, 2010); e cuidados paliativos (Benites et al., 2017). Esses estudos apontam que as pesquisas sobre a temática podem contribuir para o campo teórico e fornecer subsídios para a elaboração de propostas de intervenção em contextos desafiadores da vida.

Destacam-se, também, os conceitos positivos encontrados nos estudos, correlacionados ao sentido de vida e espiritualidade/religiosidade, a saber: a) o bem-estar (Assis et al., 2014; Barbosa et al., 2015; Estrada et al., 2012; Mello \& Araújo, 2013; Parra, 2008; Parra \& Corbetta, 2013; Payán et al., 2011); b) a qualidade de vida (Assis et al., 2014; Payán et al., 2011); c) a atitude religiosa (Aquino et al., 2009; Vieira \& Aquino, 2016), d) a resiliência (Moreira \& Holanda, 2010); e) as fontes de sentido (Damásio et al., 2013); e f) a vitalidade subjetiva (Vieira \& Aquino, 2016). Isso pode apontar o interesse de aprimoramento dos conhecimentos acerca da temática, correlacionando com outros construtos em âmbitos variados. 


\section{CONSIDERAÇÕES FINAIS}

Esta revisão integrativa da literatura teve como objetivo investigar as produções científicas em Psicologia sobre sentido de vida e espiritualidade, no contexto da América Latina. Acredita-se que a amostra das publicações encontradas pode contribuir para ampliação do conhecimento da literatura, assim como fornecer evidências acerca da temática proposta, no campo investigado. Contudo, ressalta-se que o estudo retrata apenas artigos científicos publicados nos últimos 10 anos (com exclusão de teses, dissertações, livros, resenhas e outros), utilizando como fonte de informações somente as bases de dados mencionadas, o que pode restringir o corpus da investigação. Assim, é recomendável outras investigações para avultar os conhecimentos na área.

Os resultados apresentados demonstram que existe um baixo número de publicações científicas correlacionando os construtos sentido de vida e espiritualidade no campo da Psicologia, revelando que na América Latina existe um campo de investigação a ser explorado. Conforme mencionado por Sherman e Simonton (2001) e Marques et al. (2009), foi verificado que há uma lacuna na literatura em relação aos instrumentos de mensuração do construto espiritualidade. Neste sentido, tais resultados mostram a importância de contribuições teóricas voltadas à elaboração de instrumentos psicométricos, assim como adaptação de um instrumento nacional que possa mensurar as peculiaridades culturais e regionais do contexto brasileiro.

Por fim, o presente estudo fornece elementos relevantes para trabalhos futuros acerca do sentido de vida e da espiritualidade, sendo possível a utilização de abordagens relacionadas ao bem-estar, Logoterapia e Análise Existencial e enfrentamento de circunstâncias difíceis/ desafiadoras, bem como sugere que pode ser importante correlacionar essa temática com outros conceitos positivos (qualidade de vida, atitude religiosa, fontes de sentido e vitalidade subjetiva), em contextos de vida variados (não apenas em situações difíceis da vida), demonstrando a existência de um campo científico com possibilidades de ampliação.

\section{DECLARAÇÃO DE CONFLITOS DE INTERESSE}

Os autores declaram que não há conflitos de interesse no artigo submetido.

\section{REFERÊNCIAS}

Aquino, T. A. A. de. (2009). Atitudes e Inteções de Cometer o Suicídio: seus Correlatos Existenciais e Normativos [Tese de Doutorado]. Universidade Federal da Paraíba e Universidade Federal do Rio Grande do Norte. https://docplayer.com.br/27331964-Atitudes-e-intencoes-de-cometer-o-suicidio-seus-correlatos-existenciais-e-normativos.html

Aquino, T. A. A., Correia, A. P. M., Marques, A. L. C., Souza, C. G.,Freitas, H. C. de A., Araújo, I. F., Dias, P. dos S., \& Araújo, W. F. (2009). Atitude religiosa e sentido da vida: um estudo correlacional. Psicologia: Ciência e Profissão, 29(2), 228-243. https://doi.org/10.1590/ S1414-98932009000200003

Aquino, T. A. A., Gouveia, V. V., Silva, S. S., \& Aguiar, A. A. (2013). Escala de Atitudes Religiosas, Versão Expandida (EAR-20): Evidências de validade. Avaliação Psicológica, 12(2), 109-119. http://pepsic.bvsalud.org/ pdf/avp/v12n2/v12n2a02.pdf

Assis, C. L., Faria, D. F., \& Lins, L. F. T. (2014). Bem-estar subjetivo e qualidade de vida em adeptos de ayahuasca. Psicologia \& Sociedade, 26(1), 224-234. https:// doi.org/10.1590/S0102-71822014000100024

Barbosa, S. C. U., Matamoros, F. A. S., \& Pedraza, R. S. (2015). Desarrollo de una intervención centrada en espiritualidad en pacientes con cáncer. Universitas Psychologica, 14(1), 299-311. https://doi.org/10.11144/ Javeriana.upsy14-1.dice

Barros-Oliveira, J. H. (2007). Espiritualidade e Religião: Tópicos de Psicologia Positiva. Psicologia Educação e Cultura, XI(2), 265-287.

Becker, A. P. S., \& Silva, J. D. (2016). Concepções acerca da religiosidade: a perspectiva da criança. Estudos e Pesquisas em Psicologia, 16(3), 930-952. http://www. redalyc.org/articulo.oa?id $=451854875015$

Benites, A. C., Neme, C. M. B., \& Santos, M. A. (2017). Significados da espiritualidade para pacientes com câncer em cuidados paliativos. Estudos de Psicologia (Campinas), 34(2), 269-279. https://doi.org/10.1590/198202752017000200008 
Braghetta, C. C. (2017). Desenvolvimento e validação de um instrumento para avaliar espiritualidade: Escala de Atitudes Relacionadas à Espiritualidade (ARES). Tese de Doutorado. Faculdade de Medicina da Universidade de São Paulo. http://www.teses.usp.br/teses/disponiveis/5/5142/tde-05102017-112819/en.php

Cavalheiro, C. M. F., \& Falcke, D. (2014). Espiritualidade na formação acadêmica em psicologia no Rio Grande do Sul. Estudos em Psicologia (Campinas), 31(1), 35-44. https://doi.org/10.1590/0103-166X2014000100004

Coelho, A. G. J., \& Mahfoud, M. (2001). As dimensões espiritual e religiosa da experiência humana: distinções e interrelações na obra de Viktor Frankl. Psicologia USP, 12(2), 95-103. https://doi.org/10.1590/S010365642001000200006

Coordenação de Aperfeiçoamento de Pessoal de Nível Superior. (2017). Relatório de Avaliação Quadrienal 2017:Psicologia. https://capes.gov.br/images/documentos/Relatorios_quadrienal_2017/20122017-Psicologia_relatorio-de-avaliacao-2017_final.pdf

Costa, A. B., \& Zoltowski, A. P. C. (2014). Como escrever um artigo de revisão sistemática. In S. H. Koller, M. C. P. P. Couto, \& J. V. Hohendorff (Eds.), Manual de produção científica (pp. 55-70). Penso.

Costa, C. C., Bastiani, M., Geyer, J. G., Calvetti, P. U., Muller, M. C., \& Moraes, M. L. A. (2008). Qualidade de vida e bem-estar espiritual em universitários de Psicologia. Psicologia em Estudo, 13(2), 249-255. https://doi. org/10.1590/S1413-73722008000200007

Crumbaugh, J. C., \& Maholick, L. T. (1964). An experimental study in existentialism: The psychometric approach to Frankl's concept of noogenic neurosis [Um estudo experimental em existencialismo: a abordagem psicométrica do conceito de neurose noogênica de Frankl]. Journal of Clinical Psychology, 20(1), 589-596.

Daaleman, T. P., \& Frey, B. B. (2004). The Spirituality Index of Well-Being: A New Instrument for Health-Related Quality-of-Life Research [Índice do Bem-Estar Espiritual: Um Novo Instrumento para Pesquisa de Qualidade de Vida Relacionada à Saúde]. Annals of Family Medicine, 2(5), 499-503. https://doi.org/10.1370/afm.89. Department

Damásio, B. F. (2013). Sentido de Vida e Bem-Estar Subjetivo: Interações com Esperança, Otimismo, Autoeficácia e Autoestima em Diferentes Etapas do Ciclo Vital. Tese de Doutorado. Universidade Federal do Rio Grande do Sul.
Damásio, B. F., \& Koller, S. H. (2016). Sentido de Vida: Definição e medidas. In: B. L. Seibel, M. Poletto, \& S. H. Koller (Org.), Psicologia Positiva: Teoria, pesquisa e prática (pp. 173-190). Juruá.

Damásio, B. F., Koller, S. H., \& Schnell, T. (2013). Sources of Meaning and Meaning in Life Questionnaire (SoMe): Psychometric Properties and Sociodemographic Findings in a Large Brazilian Sample [Questionário sobre fontes de significado e significado na vida (SoMe): propriedades psicométricas e achados sociodemográficos em uma grande amostra brasileira.]. Acta de Investigación Psicológica, 3(3), 1205-1228. https:// doi.org/10.1016/S2007-4719(13)70961-X

Damásio, B. F., Melo, R. L. P., \& Silva, J. P. (2013). Sentido de Vida , Bem-Estar Psicológico e Qualidade de Vida em Professores Escolares. Paidéia, 23(54), 73-82. https://doi.org/10.1590/1982-43272354201309

Ercole, F. F., Melo, L. S., \& Alcoforado, C. L. G. C. (2014). Editorial - Revisão integrativa versus revisão sistemática. Revista Mineira de Enfermagem, 18(1), 1. https:// doi.org/10.5935/1415-2762.20140001

Estrada, M. S., Fortaleza, C. G., Garcia, R. R., \& Palos, P. A. (2012). Desarrollo y evaluación psicométrica de un índice de espiritualidad para adultos mayores en México [Desenvolvimento e avaliação psicométrica de um índice de espiritualidade para idosos no México]. Psicología Iberoamericana, 20(2), 41-48. http://www. redalyc.org/pdf/1339/133928816006.pdf

Florenzano, R. U., Aspillaga, C. H., Musalem, C. A., Alliende, L., Cataldo, E., \& Arato, C. (2015). Análisis retrospectivo de las creencias religiosas en mujeres con trastorno Afectivo tratadas ambulatoriaente por riesgo suicida en un servicio de psiquiatría en Santiago de Chile [Análise retrospectiva de crenças religiosas em mulheres com transtorno afetivo e risco de suicídio, atendidas ambulatorialmente em um serviço psiquiátrico em Santiago do Chile]. Revista Argentina de Clinica Psicologica, 24(3), 223-230. http://www.redalyc. org/articulo.oa?id=281946988004\%0ACómo

Frankl, V. E. (1993). A presença ignorada de Deus. Sinodal.

Frankl, V. E. (2016). A vontade de sentido: fundamentos e aplicações da logoterapia. Paulus.

Frankl, V. E. (2018). Em busca de sentido. Vozes. 
Grouzet, F. M. E., Kasser, T., Ahuvia, A., Dols, J. M. F., Kim, Y., Lau, S., Rayn, R. M., Saunders, S., Schmuck, P. \& Sheldon, K. M. (2005). The structure of goal contents across 15 cultures [A estrutura dos Consteúdos das Metas em cerca de 15 Culturas]. Journal of Personality and Social Psychology, 89(5), 800. https://doi. org/10.1037/0022-3514.89.5.800

Gutz, L., \& Camargo, B. V. (2013). Espiritualidade entre idosos mais velhos: um estudo de representações sociais. Revista Brasileira de Geriatria e Gerontologia, 16(4), 793-804. https://doi.org/10.1590/S180998232013000400013

Koenig, H. G., King, D., \& Carson, V. B. (2012). Handbook of religion and health [Manual de religião e saúde]. Oxford University Press.

Machado, W. L, Bandeira, D. R..\& Pawlowski, J. (2013). Validação da Psychological Well-Being Scale em amostra de estudantes universitários. Avaliação Psicologica, 12 (2), 263-272. http://revistas.ufac.br/revista/ index.php/SAJEBTT/article/download/1507/1056

Marques, L. F., \& Aguiar, A. P. A. (2014). Instrumentos de mensuração da religiosidade / espiritualidade (R/E) e seus construtos. Revista Pistis Praxis, 6(427), 107. https://doi.org/10.7213/revistapistispraxis.06.001. DS06

Marques, L. F., Sarriera, J. C., \& Dell'Aglio, D. D. (2009). Adaptação e Validação da Escala de Bem-estar Espiritual (EBE). Avaliação Psicológica, 8(2), 179-186.

*Mello, M. A., \& Araújo, C. A. (2013). Velhice e espiritualidade na perspectiva da Psicologia Analítica. Boletim da Academia Paulista de Psicologia, 33(84), 118-141. http://www.redalyc.org/articulo.oa?id=94632386011

Moreira-Almeida, A., Lotufo, F. N., \& Koenig, H. G. (2006). Religiousness and mental health: A review [Religiosidade e saúde mental: uma revisão]. Revista Brasileira de Psiquiatria, 28(3), 242-250. https://doi. org/10.1590/s1516-44462006005000006

Moreira-Almeida, A., Pinsky, I., Zaleski, M., \& Laranjeira, R. (2010). Religious involvement and sociodemographic factors: a Brazilian national survey [Envolvimento religioso e fatores sociodemográficos: uma pesquisa nacional brasileira]. Revista Psiquiatria Clínica, 37(1), 1215. http://www.scielo.br/pdf/rpc/v37n1/a03v37n1

Moreira, N., \& Holanda, A. (2010). Logoterapia e o sentido do sofrimento: convergências nas dimensões espiritual e religiosa. Psico-USF, 15(3), 345-356. https:// doi.org/10.1590/S1413-82712010000300008
Núñez-Rodriguez, S. N., Souza, A. P. L., \& Koller, S. H. (2016). Cross-Cultural Adaptation and Psychometric Properties of the Portuguese Version of the Aspiration Index-AI [Adaptação transcultural e propriedades psicométricas da versão em português do Índice de Aspiração]. Temas Em Psicologia, 24(3), 1169-1180. https://doi.org/10.9788/TP2016.3-20

Oliveira, K. G., \& Aquino, T. A. A, D. (2014). A Logoterapia no contexto da Psiclologia da Religião. Interações, 9(16), 225-242. https://doi.org/10.5752/P. 1983-2478.2014v9n16p225

Oliveira, M. R. de, \& Junges, J. R. (2012). Saúde mental e espiritualidade/religiosidade: a visão de psicólogos. Estudos de Psicologia, 17(3), 469-476. https://doi. org/10.1590/S1413-294X2012000300016

Paloutzian, R., \& Ellison, C. (1982). Loneliness, spiritual well-being and the quality of life [Solidão, bem-estar espiritual e qualidade de vida]. In. L. A. Peplau \& D. Perlman (Eds.), Loneliness: A sourcebook of current theory, research and therapy (pp. 224--237). Wiley.

Pargament, K. I. (2009). The Psychology of Religion and Spirituality? Yes and No [A psicologia da religião e espiritualidade? Sim e não]. The International Journal for the Psychology of Religion, 9(1), 37-41. https://doi. org/10.1207/s15327582ijpr0901_2

Parra, A. (2008). Efectos de las Experiencias Espirituales/ Paranormales en la Vida de las Personas y su Bienestar Psicológico [Efeitos de experiências espirituais / paranormais na vida das pessoas e no bem-estar psicológico]. Revista Argentina de Clínica Psicológica, 17(3), 233-2422. http://www.redalyc.org/articulo. oa?id=281921795005\%0ACómo

Parra, A., \& Corbetta, J. M. (2013). Experiências Paranormales Y Su Relacion Con El Sentido de La Vida [Experiências paranormais e sua relação com o significado da vida]. Liberabit-Revista De Psicologia, 19(2), 251-258. http://www.redalyc.org/articulo.oa?i$\mathrm{d}=68629471011 \% 0 \mathrm{~A}$

Payán, E. C., Vinaccio, S., \& Quiceno, J. M. (2011). Cognición hacia la enfermedad, bienestar espiritual y calidad de vida en pacientes con cáncer en estado terminal [Cognição, bem-estar espiritual e qualidade de vida em pacientes com câncer terminal]. Acta Colombiana de Psicología, 14(2), 79-89. http://www.scielo.org.co/ pdf/acp/v14n2/v14n2a08.pdf 
Piedmont, R. L. (2004). Assessment of spirituality and religious sentiments: technical manual (1.a ed.). Author.

Reker, G. T., \& Wong, P. T. P. (1988). Aging as an individual process: Toward a theory of personal meaning [O envelhecimento como um processo individual: em direção a uma teoria do significado pessoal]. In J. E. Birren \& V. L. Bengtson (Eds.), Emergent theories of aging (pp. 214-246). New York, NY: Springer.

Rodrigues, A. S. G. (2015). Sentido de Vida e Suporte Social no Desenvolvimento Humano Saudável: Revisão Sistemática. Faculdade de Psicologia e de Ciências da Educação da Universidade do Porto. https://repositorio-aberto.up.pt/bitstream/10216/83287/2/119633. pdf

Santos, D. M. B. (2016). Logoterapia: Compreendendo a teoria através de mapa de conceitos. Arquivos Brasileiros de Psicologia, 68(2), 128-142. http://www.redalyc. org/articulo.oa?id=229048487011

Schnell, T. (2009). The Sources of Meaning and Meaning in Life Questionnaire (SoMe): Relations to demographics and well-being [Questionário sobre as fontes de significado e significado na vida (SoMe): relações com dados demográfics e o bem-estar]. The Journal of Positive Psychology, 4(6), 483-499.

Schnell, T., \& Becker, P. (2006). Personality and meaning in life [Personalidade e significado na vida]. Personality and Individual Differences, 41(1), 117-129. https://doi. org/10.1016/j.paid.2005.11.030

Sherman, A. C., \& Simonton, S. (2001). Assessment of religiousness and spirituality in health research [Avaliação da religiosidade e da espiritualidade na pesquisa em saúde]. In Faith and health: Psychological perspectives (pp. 139-163). Guilford Press

Silva, J. B., \& Silva, L. B.. (2014). Relação entre religião, espiritualidade e sentido da vida. Revista Logos \& Existência, 3(2), 203-215. http://periodicos.ufpb.br/ojs2/ index.php/le/article/view/22107/12148
Simkin, H. (2017). Adaptación al español de la Escala de Espiritualidad y Sentimientos Religiosos (ASPIRES): La trascendencia espiritual en el modelo de los cinco factores. Universitas Psychologica, 16(2), 1-12. https://doi.org/10.11144/Javeriana.upsy16-2

Sommerhalder, C. (2010). Sentido de Vida na Fase Adulta e Velhice. Psicologia: Reflexão e Crítica, 23(2), 270-277. https://doi.org/10.1590/S0102-79722010000200009

Steger, M. F. (2009). Meaning in life [Sentido na Vida]. In S. J. Lopez (Ed.), Oxford handbook of positive psychology (2nd ed., pp. 679-687). Oxford University Press.

Steger, M. F., Frazier, P., Oishi, S., \& Kaler, M. (2006). The meaning in life questionnaire: Assessing the presence of and search for meaning in life [Questionário sobre o significado na vida: Avaliando a presença e buscando significado na vida]. Journal of Counseling Psychology, 53(1), 80. https://doi.org/10.1037/0022-0167.53.1.80

Véras, A. D. S., \& Rocha, N. M. D. (2014). Produção de artigos sobre Logoterapia no Brasil de 1983 a 2012. Estudos e Pesquisas Em Psicologia, 14(1), 355-374. https://doi.org/10.12957/epp.2014.10483.

Vieira, D. C. R., \& Aquino, T. A. A. (2016). Vitalidade subjetiva, sentido na vida e religiosidade em idosos: um estudo correlacional. Temas Em Psicologia, 24(2), 483-494. https://doi.org/10.9788/TP2016.2-05Pt

Viktor Frankl Institut. (2018). Institutes Worldwide [Institutos em todo o mundo]. https://www.goethe.de/en/lan. html

Zinnbauer, B. J., \& Pargament, K. I. (2005). Religiousness and Spirituality [Religiosidade e Espiritualidade]. In T. G. Press (Ed.), Handbook of the psychology of religion and spirituality (pp. 21-42). Guilford Publications

Data de submissão: 13.04 .2019 Primeira decisão editorial: 25.07.2019 Aceite em 08.08.2019 